\title{
CMEARTICLE
}

\section{Narrow QRS-complex tachycardia: Part 2}

Devinder Sing ${ }^{1,2}$, MBBS, MRCP, Swee-Guan ${\underline{T e 0^{3}}}^{3}$ MRCP, FACC, Abdul Razakjr Bin $\underline{O m a r}^{2,3}, \mathrm{MRCP}, \mathrm{FACC}$, Kian-Keong $\underline{\text { Poh }}^{1,2}$, FRCP, FACC

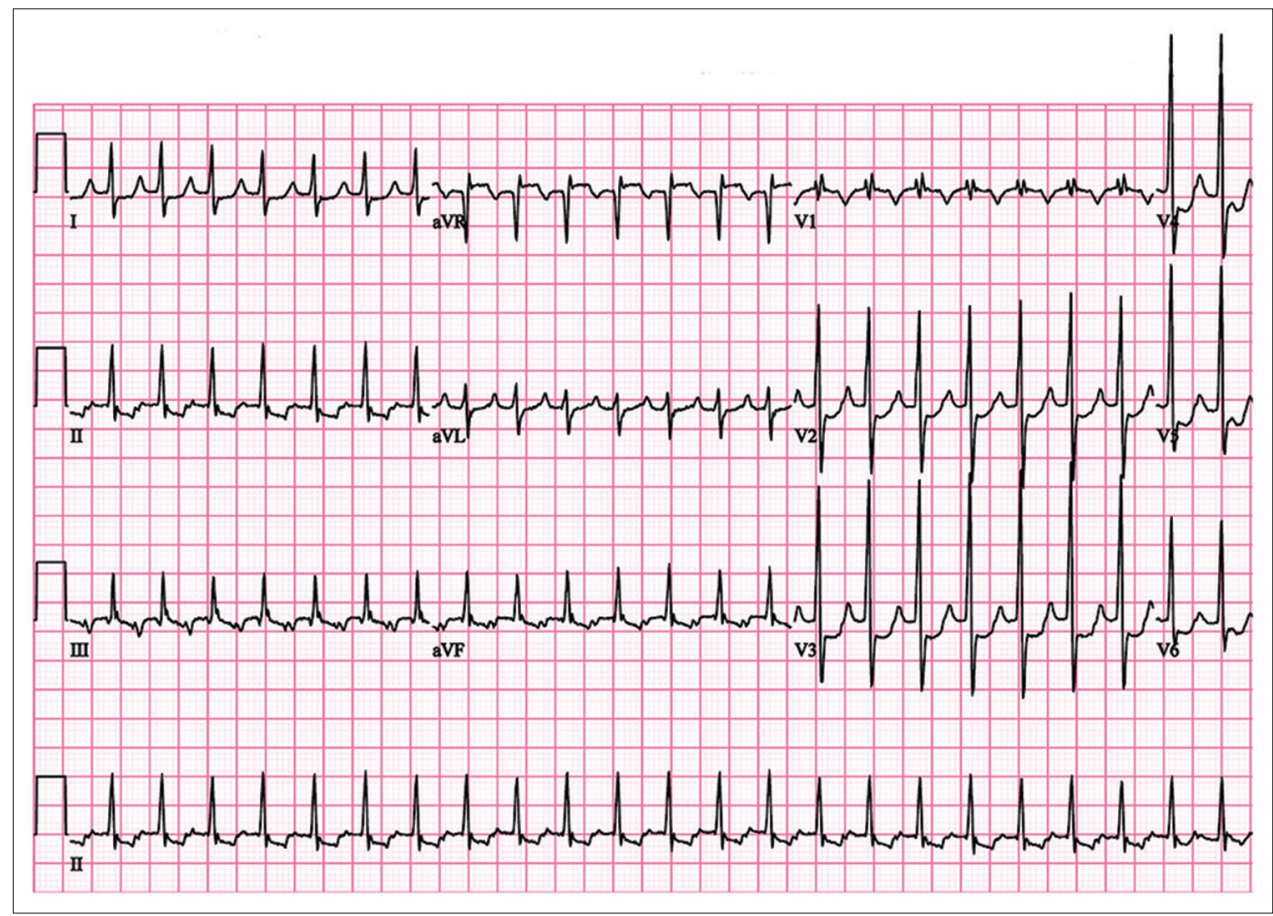

Fig. 1 ECG shows long RP narrow complex tachycardia with 1:1 AV conduction. P waves are predominantly negative in the inferior leads and lead V1.

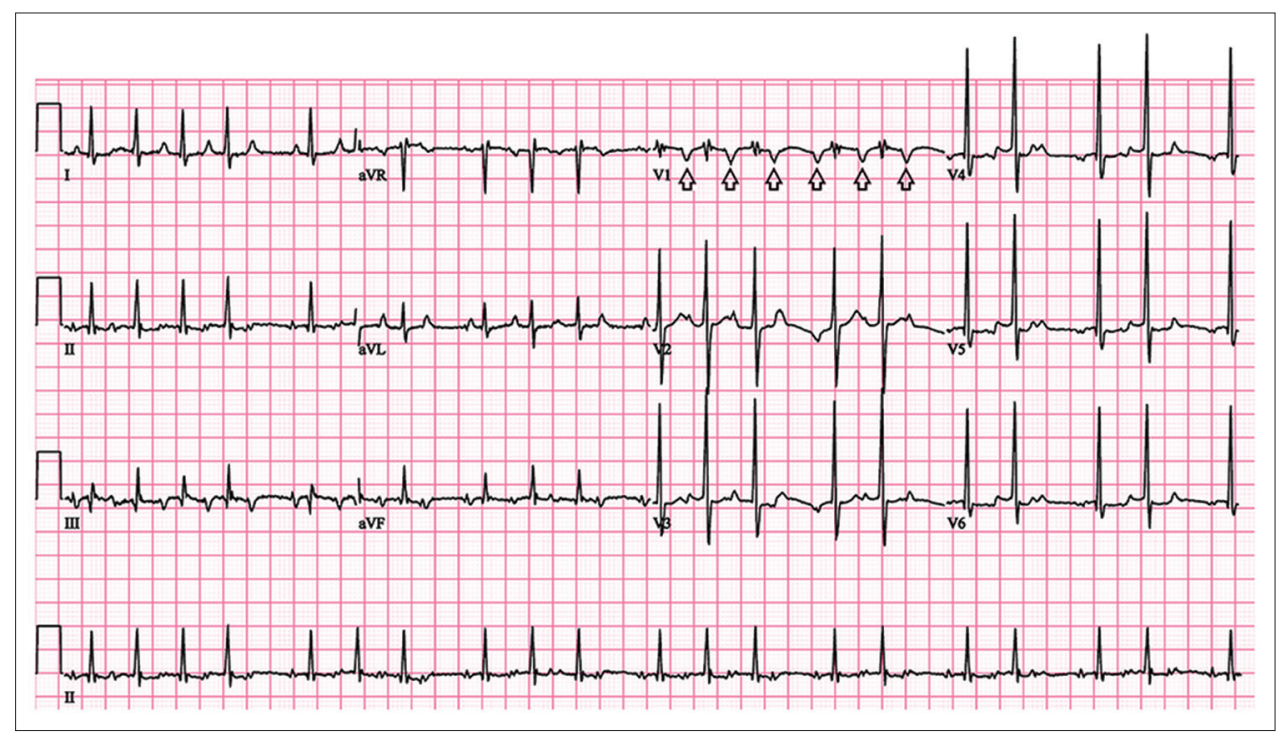

Fig. 2 ECG shows irregular narrow complex tachycardia resulting from variable degrees of the AV Wenckebach phenomenon. Arrows indicate the P waves.

\section{CASE 1}

\section{CLINICAL PRESENTATION}

A 69-year-old Malay man presented to his general physician with a history of decreased effort tolerance. He had no history of palpitation, syncope or giddiness. On clinical examination, he was tachycardic, with a heart rate of 160 beats per minute (bpm) and a blood pressure of $140 / 80 \mathrm{mmHg}$. The rest of the physical examination was unremarkable. Electrocardiography (ECG) showed regular narrow complex tachycardia at 160 bpm (Fig. 1). He was immediately referred to the emergency department.

${ }^{1}$ Department of Cardiology, National University Heart Centre, ${ }^{2}$ Yong Loo Lin School of Medicine, National University of Singapore, ${ }^{3}$ Raffles Heart Centre, Raffles Hospital, Singapore Correspondence: A/Prof Poh Kian Keong, Department of Cardiology, National University Heart Centre, 1E Kent Ridge Road, NUHS Tower Block, Level 9, Singapore 119228. kian_keong_poh@nuhs.edu.sg 


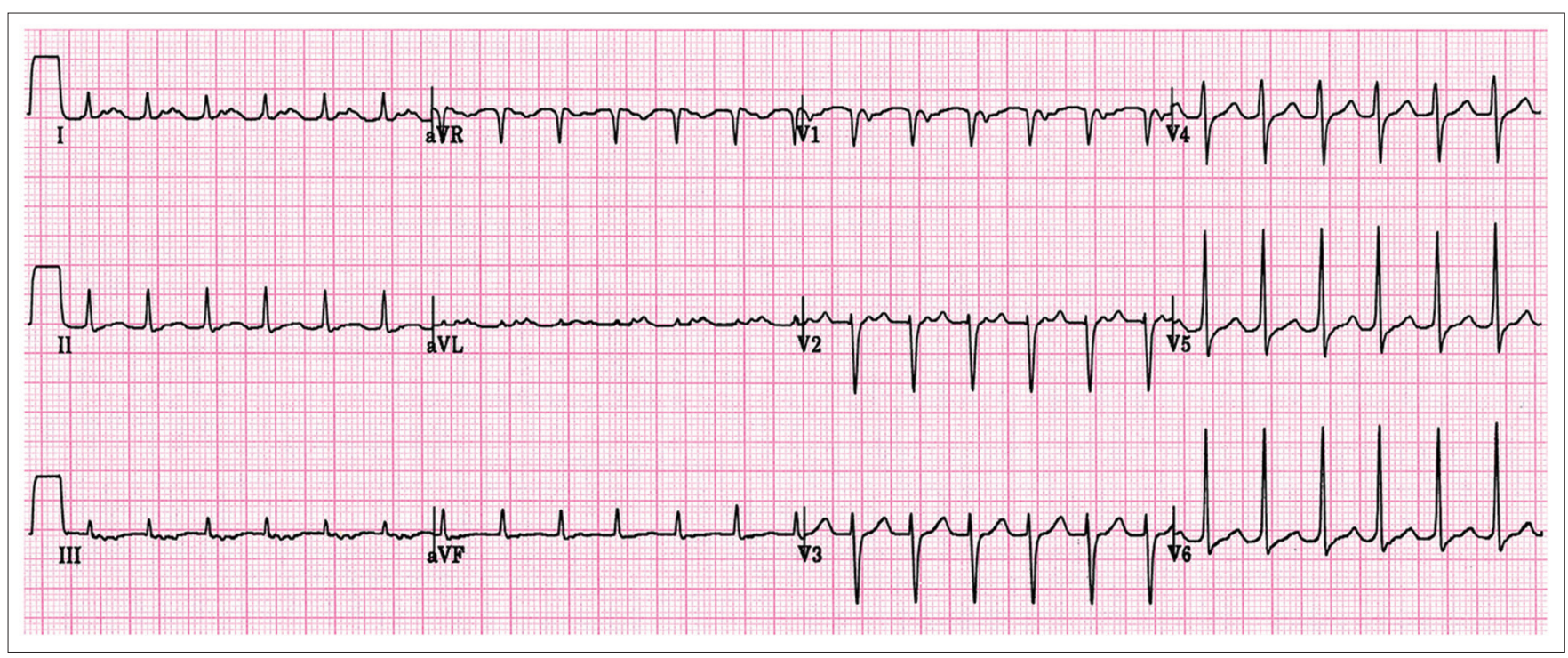

Fig. 3 ECG shows short RP regular narrow complex tachycardia, with distinct P waves seen in lead V1 following the QRS complex.

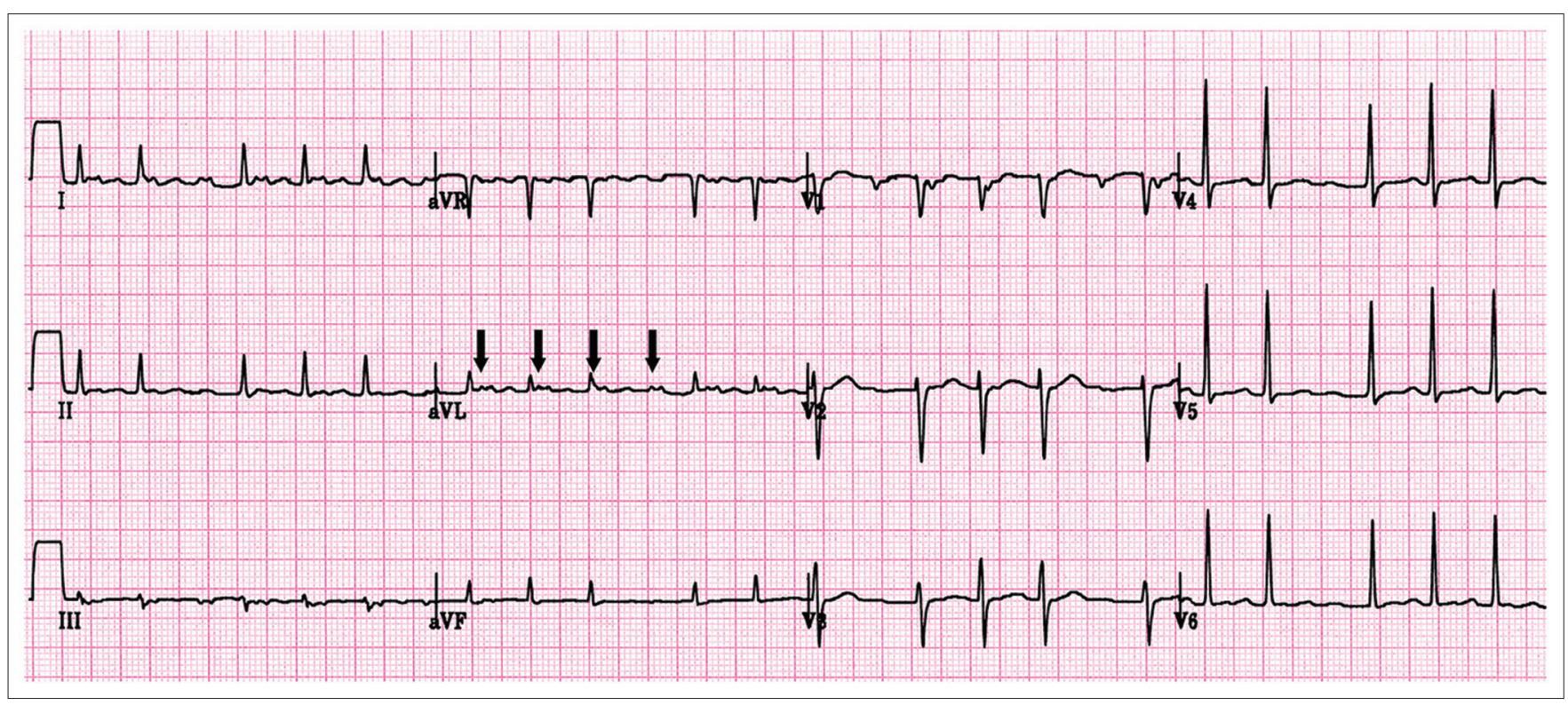

Fig. 4 ECG shows irregular narrow complex tachycardia with 'group beating' resulting from variable degrees of the AV Wenckebach phenomenon. Arrows in lead aVL indicate the P waves.

Repeat ECG showed irregular narrow complex tachycardia (Fig. 2). Tachycardia was incessant and did not respond to intravenous boluses of adenosine and verapamil. The patient was started on oral beta-blockers to slow down the ventricular response.

\section{ECG INTERPRETATION}

Initial ECG (Fig. 1) shows long RP narrow complex tachycardia with 1:1 atrioventricular (AV) conduction. $P$ waves are predominantly negative in inferior leads and lead V1. The differential diagnosis of long RP regular narrow complex tachycardia include atrial tachycardia (AT), atypical AV nodal reentrant tachycardia (AVNRT) and persistent junctional reciprocating tachycardia (PJRT), an unusual form of $\mathrm{AV}$ reentrant tachycardia (AVRT). Sinus tachycardia is also a differential for long RP tachycardia; however, the P wave axis in the ECG is not consistent with sinus $\mathrm{P}$ axis.

Subsequent ECG (Fig. 2) showed the same tachycardia as that seen in the initial ECG, with 5:4, 4:3 and 3:2 AV nodal Wenckebach phenomena. The presence of an AV block rules out AVRT or PJRT. Indeed, the above findings are consistent with that of AT.

\section{CLINICAL COURSE}

The patient continued to experience AT in spite of oral betablocker therapy. Electrophysiology confirmed focal AT arising from the anterior right atrium near the tricuspid annulus. Radiofrequency ablation at the point of the earliest activation led to the termination of tachycardia, and the patient remained in sinus rhythm thereafter. 


\section{CASE 2}

\section{CLINICAL PRESENTATION}

A 67-year-old Chinese woman presented with a two-month history of palpitation associated with shortness of breath. She used to experience multiple daily episodes of palpitations that lasted from minutes to hours. There was no accompanying history of chest pain, syncope or giddiness. Physical examination was unremarkable, except for a resting heart rate of $150 \mathrm{bpm}$. Clinically, the patient was not having heart failure. ECG (Fig. 3) showed regular narrow complex tachycardia at a rate of $150 \mathrm{bpm}$. The patient was administered repeated boluses of intravenous adenosine and diltiazem, with no termination of tachycardia. However, there was a slowing of the heart rate following the administration of diltiazem, and ECG at that time showed irregular rhythm (Fig. 4).

\section{ECG INTERPRETATION}

Initial ECG (Fig. 3) shows short RP regular narrow complex tachycardia, with a distinct $\mathrm{P}$ wave seen in lead V1 following the QRS complex. The differential diagnoses of short RP tachycardia include typical AVNRT, AVRT and AT. However, the slowing of $\mathrm{AV}$ nodal conduction after intravenous administration of diltiazem narrowed down the differential diagnoses. Fig. 4 shows the continuation of tachycardia with a 3:2 AV node Wenckebach phenomenon (arrows pointing toward the P wave). Continuation of tachycardia in spite of an AV node block rules out AVRT. AT is the most likely diagnosis given the finding of a 3:2 AV nodal conduction.

\section{CLINICAL COURSE}

The patient was admitted and put on telemetry for monitoring. Oral beta-blocker was started to slow down AV nodal conduction and to control the ventricular rate. Telemetry monitoring showed incessant AT; it rarely terminated for few beats and restarted again. Echocardiography showed moderately impaired left ventricular systolic function with an ejection fraction of $40 \%$. Coronary angiogram revealed normal coronaries. Electrophysiology confirmed the presence of focal AT arising from a low crista terminalis in the right atrium. Radiofrequency ablation of AT was successfully performed, with restoration of the patient's sinus rhythm. The patient was advised follow-up echocardiography to assess any improvement in left ventricular ejection fraction following ablation.

\section{DISCUSSION}

AT is a regular atrial rhythm at a rate greater than $100 \mathrm{bpm}$ originating from outside of the sinus node. (1) AT mostly presents as regular narrow complex tachycardia. ${ }^{(2)}$ The major causes of regular narrow complex tachycardia are AVNRT, AVRT, AT and sinoatrial node reentrant tachycardia (SNRT). AVNRT accounts for approximately $60 \%$ of cases, AVRT for approximately $30 \%$, and SNRT and AT for $10 \%$ of cases. ${ }^{(3)}$ In contrast to atrial flutter, which is a macroreentrant tachycardia involving a large circuit, AT arises from a single site in either the right or left atrium. Focal AT is mostly paroxysmal and self-limiting, but may present as incessant AT associated with left ventricular dysfunction. ${ }^{(4)}$ Focal AT is relatively uncommon, accounting for $5 \%-15 \%$ of arrhythmias in adults who undergo diagnostic studies of paroxysmal supraventricular tachycardia. ${ }^{(5)}$ AT can be produced by three distinct electrophysiologic mechanisms, namely enhanced automaticity, triggered activity and microreentry. Focal AT arises more commonly from the right atrium. ${ }^{(6)}$ The most common clinical presentation is paroxysmal palpitations associated with episodes of AT. Rarely, it can also present as syncope if ventricular rate exceeds $180 \mathrm{bpm}$. Conditions associated with focal AT include hypertension, cardiomyopathy, pulmonary decompensation, infection, excessive alcohol intake, hypokalaemia, hypoxia, stimulants, cocaine ingestion and theophylline therapy. ${ }^{(7)}$

Atrial rate during focal AT is usually about 130-250 bpm. The $\mathrm{P}$ wave morphology can either appear normal or abnormal depending on the site of origin. Although focal AT is regular, the rate may accelerate or 'warm up' in the first few beats and decelerate in the last few beats. AT is not dependent on AV nodal conduction, unlike AVRT. Therefore, the presence of an AV block, especially a Wenckebach phenomenon with the appearance of 'grouped beating', strongly favours AT, as shown in the two clinical cases described earlier. Although algorithms based on the morphology of P wave to localise AT have been described, they are beyond the scope of this article. ${ }^{(4)}$

Acute management of AT is guided by the symptoms, ventricular rate and haemodynamic stability of the patient. Efforts should be made to correct any underlying precipitants such as digitalis toxicity or electrolyte imbalance. Intravenous adenosine, beta-blockers and non-dihydropyridine calcium channel blockers may be used to terminate AT. Apart from terminating AT, AV nodal blocking drugs may help to differentiate AT from AVNRT and AVRT. Patients with haemodynamically unstable AT should be treated with electrical cardioversion. Chronic therapy is useful for prevention of recurrence in patients with repetitive episodes of focal AT. Oral beta-blockers and non-dihydropyridine calcium channel blockers (diltiazem or verapamil) are recommended for initial therapy. In patients with recurrent AT despite therapy, electrophysiology studies with ablation should be considered, as described in the earlier case vignettes where both patients presented with incessant AT that was unresponsive to drugs. In such a scenario, electrophysiology study with radiofrequency ablation is the treatment of choice.

ABSTRACT We discuss two cases of incessant atrial tachycardia (AT), including the presentation and clinical course. It is important to differentiate AT from other causes of supraventricular tachycardia, such as atrioventricular nodal reentrant tachycardia (AVNRT) and atrioventricular reentrant tachycardia (AVRT), as it would have implications on clinical management. Electrocardiographic features of AT, especially the presence of an AV Wenckebach phenomenon with 'grouped beating', are critical for differentiating AT from AVRT and AVNRT. It is also vital to identify the $P$ waves and their relations to $Q R S$ on electrocardiography, as this would aid in the differentiation of various supraventricular tachycardias.

Keywords: atrial tachycardia, atrioventricular Wenckebach phenomenon, palpitations, regular narrow complex tachycardia 


\section{REFERENCES}

1. Saoudi N, Cosío F, Waldo A, et al. A classification of atrial flutter and regular atrial tachycardia according to electrophysiological mechanisms and anatomical bases; a Statement from a Joint Expert Group from The Working Group of Arrhythmias of the European Society of Cardiology and the North American Society of Pacing and Electrophysiology. Eur Heart J $2001 ; 22: 1162-82$.

2. Singh D, Teo SG, Poh KK. Electrocardiography Series. Regular narrow complex tachycardia. Singapore Medical J 2011; 52:146-9; quiz 450.

3. Blomström-Lundqvist, C, Scheinman, MM, Aliot, EM, et al. ACC/AHA ESC guidelines for the management of patients with supraventricular arrhythmias-executive summary: a report of the American College of Cardiology/American Heart Association Task Force on Practice Guidelines and the European Society of Cardiology Committee for Practice Guidelines (Writing Committee to Develop Guidelines for the Management of Patients
With Supraventricular Arrhythmias). Circulation 2003; 108:1871-909.

4. Medi C, Kalman JM, Haqqani H, et al. Tachycardia-mediated cardiomyopathy secondary to focal atrial tachycardia: long-term outcome after catheter ablation. J Am Coll Cardiol 2009; 53:1791-7.

5. Chen SA, Chiang CE, Yang CJ, et al. Sustained atrial tachycardia in adult patients. Electrophysiological characteristics, pharmacological response, possible mechanisms, and effects of radiofrequency ablation. Circulation 1994; 90:1262-78.

6. Kistler PM, Roberts-Thomson KC, Haqqani HM, et al. P-wave morphology in focal atrial tachycardia: development of an algorithm to predict the anatomic site of origin. J Am Coll Cardiol 2006; 48:1010-7

7. Akhtar M. Supraventricular tachycardias. Electrophysiologic mechanisms, diagnosis and pharmacologic therapy. In: Josephson ME, Wellens $\mathrm{H}$, eds. Tachycardias: Mechanisms, Diagnosis, Treatment. Philadelphia: Lea \& Febiger, 1984: 137-69. 


\section{SINGAPORE MEDICAL COUNCIL CATEGORY 3B CME PROGRAMME} (Code SMJ 201409A)

1. Regarding focal atrial tachycardia (AT):

(a) It is the most common cause of supraventricular tachycardia.

(b) It arises more commonly from the right atrium than the left atrium.

(c) Incessant AT may lead to left ventricular systolic dysfunction.

(d) Palpitation is the most common presenting symptom of AT.

2. The following conditions are associated with focal AT:

(a) Hypertension.

(b) Cardiomyopathy.

(c) Pulmonary decompensation.

(d) Excessive alcohol ingestion.

3. Regarding the electrocardiographic features of focal AT:

(a) Atrial rate during focal AT is usually 130-250 bpm.

(b) AT is dependent on atrioventricular (AV) nodal conduction.

(c) The presence of an AV Wenckebach phenomenon during supraventricular tachycardia differentiates AT from atrioventricular reentrant tachycardia (AVRT).

(d) 'Group beating' during supraventricular tachycardia strongly favours AT.

4. The following are possible eletrophysiologic mechanisms associated with focal AT:

(a) Automaticity.

(b) Micro-reentry.

(c) Triggered activity.

(d) Macro-reentry.

5. Regarding the management of AT:

(a) AV nodal blocking agents may help differentiate AT from AVRT.

(b) Haemodynamically unstable AT should be treated with electrical cardioversion.

(c) Oral beta-blockers and calcium channel blockers can be used to prevent recurrence of AT.

(d) Electrophysiology with radiofrequency ablation is an option for the management of AT, especially incessant AT.

\section{Doctor's particulars:}

Name in full

MCR number

Email address

\section{SUBMISSION INSTRUCTIONS:}

(1) Log on at the SMJ website: http://www.sma.org.sg/publications/smjcurrentissue.aspx and select the appropriate set of questions. (2) Provide your name, email address and MCR number. (3) Select your answers and click "Submit".

\section{RESULTS:}

(1) Answers will be published in the SMJ November 2014 issue. (2) The MCR numbers of successful candidates will be posted online at the SMJ website by 6 November 2014 . (3) Passing mark is $60 \%$. No mark will be deducted for incorrect answers. (4) The SMJ editorial office will submit the list of successful candidates to the Singapore Medical Council. (5) One CME point is awarded for successful candidates.

Deadline for submission: (September 2014 SMJ 3B CME programme): 12 noon, 30 October 2014. 